\title{
Aprovechamiento de subproductos de la faena de aves. Curtido de pieles de pata de gallina
}

\section{A profitable use of by-products of the poultry slaughter. The tanning of hen's feet skins}

\author{
Iade, Juan (1), Casanova, Susana ${ }^{(2)}$, Losada, Leticia ${ }^{(3)}$, Larrosa, Andrés ${ }^{(3)}$ \\ ${ }^{(1)}$ Departamento de Textiles, Lana Bruta, Tops y Cueros, LATU, Uruguay - (2) Área de Patología y Producción Avícola, \\ Facultad de Veterinaria, Universidad de la República, Uruguay - ${ }^{(3)}$ Facultad de Veterinaria, Universidad de la República, \\ Uruguay.
}

Contacto: jiade@latu.org.uy

Recibido: 15/6/2012 - Aprobado: 27/9/2012

\begin{abstract}
Resumen
El objetivo de este trabajo es lograr generar valor agregado a uno de los subproductos de la faena de aves, las patas de gallina. Se partió de pieles extraídas de patas de gallina a las que se les realizó un proceso de curtido para obtener un cuero caracterizado por su aspecto similar al de cueros exóticos, como los de reptil. Para facilitar su posible producción por medios artesanales, se diseñó un proceso más amigable con el medio ambiente, eliminando el uso de sulfuro de sodio en la etapa correspondiente al pelambre y encalado, seguido de un curtido libre de cromo basado en una combinación de taninos vegetales y recurtientes sintéticos. La confirmación del curtido de las pieles se realizó midiendo la temperatura de contracción de los cueros, cuyo valor fue de $80^{\circ} \mathrm{C}$, superior al de las pieles crudas. Se evaluaron algunas propiedades físicas para caracterizar el producto y se obtuvieron valores de resistencia a la tracción de $0,65 \mathrm{~kg} / \mathrm{mm}^{2}$ y de resistencia al desgarro de $2,10 \mathrm{~kg} / \mathrm{mm}$.

Palabras clave: Cuero, pollo, curtido.
\end{abstract}

\begin{abstract}
$\underline{\text { Abstract }}$
The aim of this development is to add value to one of the by-products of the poultry slaughtery, the chicken's feet. The process began with the skinning of the chicken's feet, which underwent a tanning process to obtain a leather characterised for its similarity with exotic leathers such as the reptile ones. To facilitate its possible production by artisanal means we designed a more environmental-friendly process, eliminating the use of sodium sulfide in the stages corresponding to dehairing and liming, followed by a chromium-free tanning based on a combination of vegetable tannins and synthetic retanning agents. Confirmation of the tanning of the skins was performed by measuring the shrinkage temperature of leathers, obtaining a value of $80{ }^{\circ} \mathrm{C}$, higher than those of the raw hides. Physical properties were evaluated to characterize the product, obtaining tensile strength values of $0,65 \mathrm{~kg} / \mathrm{mm}^{2}$ and a tear resistance of $2,10 \mathrm{~kg} / \mathrm{mm}$.

Keywords: Leather, chicken, tanned.
\end{abstract}

\section{Introducción}

La faena de aves para su consumo genera una variedad de subproductos con poco o ningún valor agregado, entre ellos, las patas de gallinas. En Uruguay estas son utilizadas para la fabricación de harinas de carne con destino a la alimentación animal, o en pequeña escala para su exportación como alimento a países del sudeste asiático. Para ser exportadas como alimento son lavadas, escaldadas, peladas y clasificadas para su posterior congelamiento. Durante la faena de las aves las patas son separadas de la canal y utilizadas en la fabricación de harina de reciclado, junto con plumas, vísceras no comestibles y otras proteínas, como restos de carne. Estas harinas se utilizan principalmente para alimentación de animales de compañía. Las patas están exentas de plumas y se encuentran cubiertas por escamas reptilianas que no se superponen entre sí y están compuestas de queratina. Estas escamas se clasifican en cuatro tipos: unas muy pequeñas, llamadas Cancela, que son un engrosamiento de la piel, las Retículas, que aparecen en los laterales del metatarso, formadas por alfa queratina, las Escutas, que son las más grandes, aparecen en la parte anterior del metatarso y están formadas por beta queratina, y las Escutelas, no tan grandes como las Escutas, ubicadas en la parte posterior del metatarso.

Como toda piel de vertebrados, la piel de las patas de gallina tiene colágeno en su constitución. Posee epidermis, dermis e hipodermis y no presenta glándulas epiteliales.

Por su contenido de colágeno esta piel es factible de ser sometida a un proceso de curtido para obtener cuero, un producto con mayor valor agregado que las harinas.

Pese a su pequeño tamaño, su valor radica en el hecho de permitir la confección de productos de cuero que presentan el aspecto característico de las pieles de reptil, determinado por el diseño de las escamas. 
Para el curtido de una piel en general, son muchas las opciones de procesamiento por las que se puede optar en cada una de las etapas.

Una visión esquemática del proceso de un cuero abarca las siguientes fases: remojo, lavado, pelambre, encalado, desencalado, purga, piquelado, curtido, recurtido, engrase, teñido y terminación. Los procesos de recurtido, engrase y teñido se pueden realizar de uno a la vez, o combinándolos entre sí, hasta en un solo tratamiento.

De estas etapas, la de pelambre y la de curtido son las que presentan más dificultades ambientales, debido a que los efluentes que se generan requieren su procesamiento para no producir contaminación.

En el caso de las pieles de pata de gallina, una de las etapas que se puede eliminar es la del pelambre por la ausencia de pelo, evitando el uso del sulfuro de sodio, uno de los productos más contaminantes en el proceso de curtido.

Otro paso en el que se puede lograr un proceso más amigable con el medio ambiente es la etapa de curtido, prescindiendo del uso de sales de cromo y sustituyéndolo por taninos vegetales. La mayoría de los cueros que se producen en el mundo, $80 \%$ a $85 \%$, se curten con cromo, debido a que los otros curtidos no pueden competir en cuanto a las propiedades que le otorgan a los cueros y a los costos de producción. Sin embargo, hay un mayor interés en el mundo por desarrollar curtidos libres de cromo, dado por las presiones a favor de mejores resultados ambientales.

Para este proceso de las pieles de pata de gallina se optó por un curtido realizado con curtientes vegetales, libre de metales.

\section{Materiales y Métodos}

\section{Procesamiento de cuero de pata de pollo}

Las patas de gallina utilizadas son de reproductoras pesadas, de la línea Cobb, que se envían a faena al final de su vida reproductiva. Las patas se obtuvieron en una planta de faena y se conservaron congeladas hasta el momento en que se comenzaron a procesar. Para empezar el proceso se descongelaron a temperatura ambiente y luego se desollaron para obtener la piel. Para hacerlo se utilizó un bisturí y esta fue la etapa más engorrosa y lenta del proceso.

El procesamiento de las pieles se realizó en un fulón de laboratorio, de acero inoxidable, marca Otto Spech, con controles de velocidad de giro, tiempo de marcha y parada del tambor, así como control de temperatura del baño. Los productos químicos utilizados son los habituales en una curtiembre de calidad comercial, y se toman como composición y propiedades las que declara el fabricante.

El proceso de curtido de las pieles se presenta esquemáticamente en la Tabla 1, que indica los tiempos de marcha y detención del tambor del fulón, las cantidades de productos agregados, en qué momento adicionarlos, $\mathrm{pH}$ y temperatura del baño, así como distintas acciones mecánicas realizadas a lo largo del proceso.

Se comenzó con un lavado de las pieles con agua y tensoactivo no iónico, para empezar a eliminar resto de carne, grasa, sangre y suciedad de la piel.

Dado que se descartó la etapa de pelambre, se pasó directamente al proceso de encalado, donde en un nuevo baño de agua se agregó cal para lograr la apertura de las fibras del colágeno de la piel, eliminar proteínas globulares, grasa y fundamentalmente las escamas reptilianas de queratina, que le dan rigidez a la piel de la pata de pollo.

Tras un baño de encalado por 48 horas, las pieles se retiraron y se procedió a un tratamiento mecánico, raspando el lado carne de las pieles y las escamas con el lomo de una cuchilla, para poder eliminar tanto restos de grasa y carne como las escamas.

Con lavados con agua y tensoactivo no iónico se eliminó la cal no combinada con la piel y restos de carne, grasa y escamas.

Para eliminar la cal unida químicamente a la piel se lavaron las pieles en un baño de agua, con el agregado de sulfato de amonio y cloruro de amonio.

Luego se pasó a la etapa de rendido, donde en un baño de agua, a una temperatura de $37^{\circ} \mathrm{C}$ y un $\mathrm{pH}$ aproximado a 8 , se agregó una purga enzimática, de actividad aproximada a $3000 \mathrm{LEV} / \mathrm{g}$. Las purgas cumplen la función de abrir las fibras del colágeno por medio de una peptización controlada, a la vez que contribuyen a la limpieza de la piel y a revertir el hinchamiento alcalino de la etapa de encalado. Fue necesario repetir la purga en algún batch de esta etapa del proceso, en que las pieles no estaban lo suficientemente rendidas. El control de la efectividad del rendido o purga se realizó presionando el lado flor con el dedo; cuando la impresión digital quedó marcada en la piel, se consideró que la purga fue satisfactoria, en caso contrario, se repitió esta etapa.

En estas condiciones las pieles estuvieron prontas para entrar a las etapas de piquel y curtido.

Se preparó entonces el baño de piquelado. Para realizar un curtido vegetal, el pH del baño de piquel debe ser de aproximadamente 5, de manera de permitir el ingreso de los taninos a la piel. Con el fin de evitar el hinchamiento ácido de las pieles, lo que dificultaría el ingreso de los productos a la piel, se le agregó cloruro de sodio al baño de agua, previo al agregado de ácido fórmico.

Una vez que el baño de piquel alcanzó el pH 5, se verificó que en el interior las pieles estuviesen al mismo $\mathrm{pH}$, aproximadamente. Para esto, en un corte de la piel se agregó una gota de verde de bromocresol, que cambió a un color verde azulado, el cual indica que el $\mathrm{pH}$ era adecuado.

Se adicionó al baño de piquelado un auxiliar de curtición para facilitar el ingreso a la piel de los agregados de taninos vegetales. Los taninos utilizados fueron extracto de mimosa y extracto de quebracho; primero se incorporó la mimosa, en cantidades crecientes y en intervalos de tiempo de acuerdo a la Tabla 1. Por último, se agregó el quebracho.

Para fijar los taninos al colágeno se bajó el $\mathrm{pH}$ con ácido fórmico hasta un valor aproximado a 3,8. Una vez alcanzado este $\mathrm{pH}$, se descartó el baño y se dejaron descansar los cueros en un caballete toda la noche.

Terminada la etapa de curtido y transformadas las pieles en cuero, se pasó a la etapa de recurtido, en la que se le otorgan al cuero las propiedades de blandura, suavidad, elasticidad y resistencia al desgarro y tracción.

En un nuevo baño de agua, con el agregado de un auxiliar de curtición, se realizó el recurtido con extracto de quebracho más el agregado de un recurtiente sintético de sustitución, buscando con este último dar un efecto de relleno del cuero y suavidad al tacto. En el mismo proceso se procedió al engrase, para lo que se utilizaron tres tipos de aceites para otorgarle la blandura necesaria al cuero y mejorar otras propiedades, como la tracción o el desgarro: un aceite sintético, un aceite de pata de vaca y un aceite en base a aceite de pescado sulfitado y aceite sintético. El engrase le da a los cueros las propiedades de blandura, buena elasticidad y una mayor resistencia al desgarro.

Para fijar estos productos del recurtido al cuero se agregó ácido fórmico, y se llegó a un $\mathrm{pH} 3,5-3,8$.

Una vez terminado el recurtido y engrase se pasó a la etapa de teñido, en la que se tiñeron los cueros de dos colores distintos, por lo que se dispuso un nuevo baño de agua para cada color. Se realizó un agregado de formiato de sodio para que los baños alcanzaran un valor de $\mathrm{pH} 4,8$, de manera que los cueros estuvieran en condiciones de permitir la penetración de los colorantes. Se agregaron los colorantes y verificada su penetración en los cueros se procedió a su fijación, para lo que se agregó ácido fórmico hasta un $\mathrm{pH} 3,8$ y se elevó la temperatura del baño a $40{ }^{\circ} \mathrm{C}$. Finalmente, se adicionó un engrase catiónico a base de aceites naturales y un agregado de ácido fórmico.

Una vez culminado el teñido se dejaron secar los cueros al aire, se ablandaron manualmente y se les dio una terminación en base a la aplicación de una cera natural.

En la Figura 1 se muestran los cueros obtenidos. 


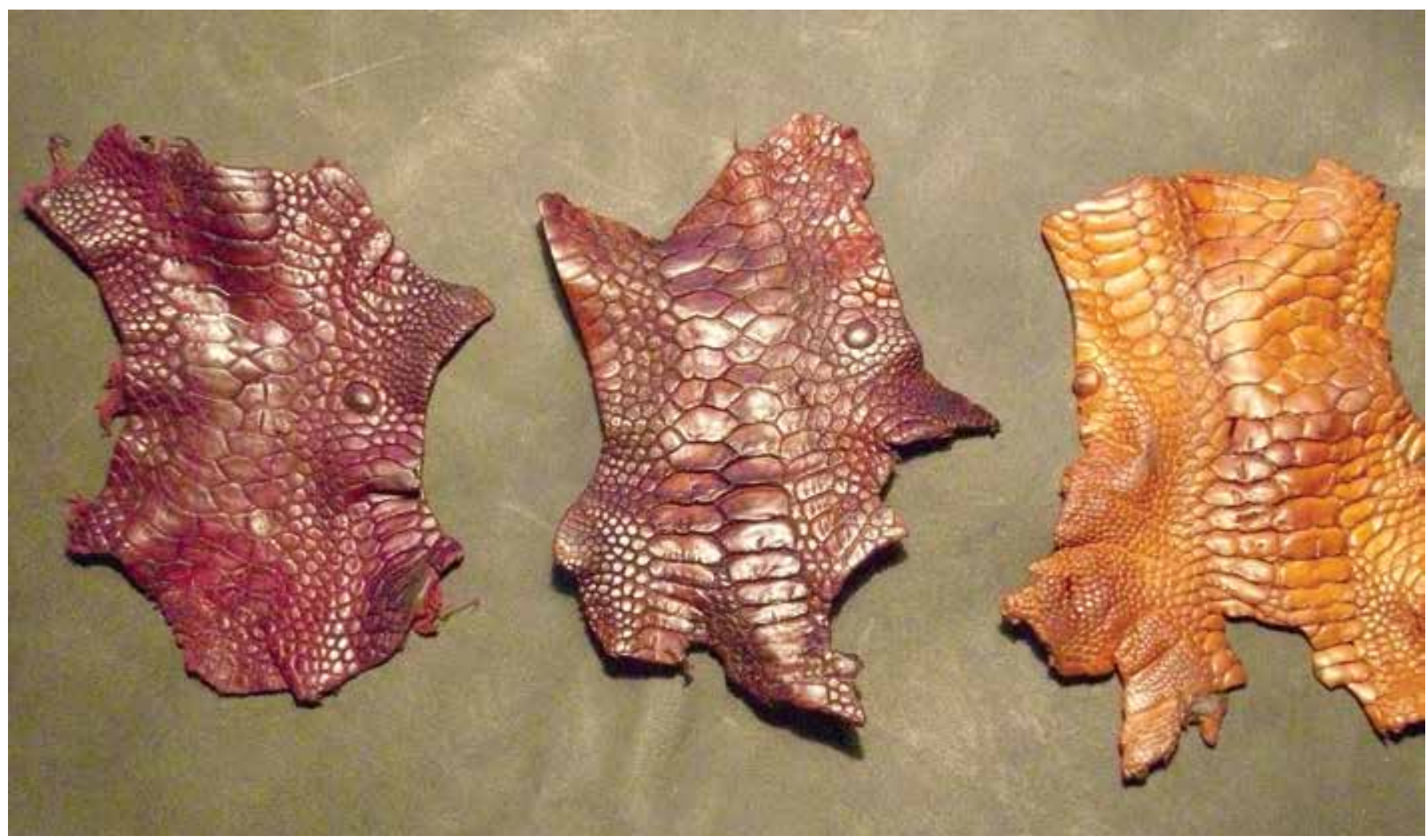

Figura 1. Cueros de pata de gallina.

\begin{tabular}{|c|c|c|c|c|c|}
\hline Proceso & Producto & $\begin{array}{l}\text { Porcentaje de producto } \\
\text { referido a peso de la piel } \\
\text { entrar al proceso }\end{array}$ & $\begin{array}{l}\text { Temperatura } \\
\text { del baño }\end{array}$ & $\begin{array}{l}\text { Movimiento del fulón } \\
\text { /Observaciones }\end{array}$ & \\
\hline \multirow[t]{3}{*}{ LAVADO } & Agua & $150,00 \%$ & & & \\
\hline & Tensoactivo no iónico & $0,50 \%$ & & & \\
\hline & Bactericida & $0,20 \%$ & & Mueve 1 hora & Tirar baño \\
\hline \multirow[t]{6}{*}{ ENCALADO } & Agua & $200,00 \%$ & & & \\
\hline & Cal & $1,00 \%$ & & Mueve 1 hora & \\
\hline & Cal & $1,00 \%$ & & Mueve 1 hora & \\
\hline & & & & Mueve 5' / hora durante 48 horas & \\
\hline & & & & Tirar baño & \\
\hline & & & & Descarnar y eliminar escamas & \\
\hline \multirow[t]{2}{*}{ LAVADO } & Agua & $100,00 \%$ & & 10 & Tirar baño \\
\hline & Agua & $100,00 \%$ & & 20 & Tirar baño \\
\hline \multirow[t]{4}{*}{ DESENCALADO } & Agua & $150,00 \%$ & & & \\
\hline & NH4CI & $0,50 \%$ & & Mueve 1 hora & \\
\hline & (NH4)2SO4 & $0,50 \%$ & & Mueve 1 hora & \\
\hline & & & & Tirar baño & \\
\hline \multirow[t]{4}{*}{ PURGA } & Agua & $100,00 \%$ & $37^{\circ} \mathrm{C}$ & $\mathrm{pH}=8$ & \\
\hline & Tensoactivo & $0,50 \%$ & & Mueve 5' & \\
\hline & Purga Enzimática & $0,25 \%$ & & Mueve 1 hora & \\
\hline & & & & $\begin{array}{c}\text { Verificar buen purgado } \\
\text { (se debió repetir la purga } \\
\text { en algunas pieles) }\end{array}$ & \\
\hline
\end{tabular}




\begin{tabular}{|c|c|c|c|c|c|}
\hline Proceso & Producto & $\begin{array}{l}\text { Porcentaje de producto } \\
\text { referido a peso de la piel } \\
\text { entrar al proceso }\end{array}$ & $\begin{array}{c}\text { Temperatura } \\
\text { del baño }\end{array}$ & $\begin{array}{c}\text { Movimiento del fuló } \\
\text { /Observaciones }\end{array}$ & \\
\hline \multirow[t]{4}{*}{ PIQUELADO } & Agua & $100,00 \%$ & & & \\
\hline & Sal & $8,0 \%$ & & Mueve 10' & \\
\hline & Ac. Fórmico & $\mathbf{0 , 5 0} \%$ & & Mueve 20' & \\
\hline & Ac. Fórmico & $0,50 \%$ & & Mueve 30' $\mathrm{pH}=5$ & \\
\hline \multirow[t]{10}{*}{ CURTIDO } & Agregar al baño de piquelado & & & & \\
\hline & Auxiliar de curtido & $2,00 \%$ & & Mueve 15' & \\
\hline & Extracto de mimosa & $2,00 \%$ & & Mueve 1 hora & \\
\hline & Extracto de mimosa & $5,00 \%$ & & Mueve 1 hora & \\
\hline & Extracto de mimosa & $10,00 \%$ & & Mueve 2 horas & \\
\hline & Extracto Quebracho & $5,00 \%$ & & Mueve 2 horas & \\
\hline & & & & Mueve 5' / hora toda la noche & \\
\hline & Ac. Fórmico & $1,00 \%$ & & Mueve 1 hora $\mathrm{pH}=3,8$ & \\
\hline & & & & Descargar & \\
\hline & & & & $\begin{array}{l}\text { Descansan cueros } \\
\text { toda la noche }\end{array}$ & \\
\hline \multirow[t]{9}{*}{ RECURTIDO } & Agua & $200,00 \%$ & & & \\
\hline & Auxiliar de curtido & $1,00 \%$ & & Mueve 15' & \\
\hline & Extracto Quebracho & $5,00 \%$ & & & \\
\hline & Aceite sintético & $2,00 \%$ & & Mueve 1 hora & \\
\hline & $\begin{array}{c}\text { Recurtiente sintético } \\
\text { de sustitución }\end{array}$ & $2,00 \%$ & & Mueve 1 hora & \\
\hline & Aceite de patas crudo & $4,00 \%$ & & Mueve 10' & \\
\hline & $\begin{array}{l}\text { Aceite de pescado sulfitado } \\
\text { y aceites sintéticos }\end{array}$ & $6,00 \%$ & & Mueve 1 hora & \\
\hline & Ac. Fórmico & $1,00 \%$ & & Mueve 1 hora $\mathrm{pH}=3,5-3,8$ & \\
\hline & & & & Descargar & \\
\hline \multirow[t]{9}{*}{ TEÑIDO } & Agua & $80,00 \%$ & & & \\
\hline & Formiato de sodio & $0,25 \%$ & & Mueve 15' $\mathrm{pH}=4,8$ & \\
\hline & Colorante & $3,00 \%$ & & Mueve 20' & \\
\hline & Ac. Fórmico & $1,00 \%$ & & Mueve 15' $\mathrm{pH}=3,8$ & \\
\hline & & & $30^{\circ} \mathrm{C}$ & Mueve 20' & \\
\hline & & & $40{ }^{\circ} \mathrm{C}$ & Mueve 20' & \\
\hline & Aceite catiónico & $1,00 \%$ & & Mueve 20' & \\
\hline & Ac. Fórmico & $1,00 \%$ & & Mueve 20' & \\
\hline & & & & Tirar baño & \\
\hline \multirow[t]{2}{*}{ ENJUAGAR } & Agua & $100,00 \%$ & & Mueve 15, & \\
\hline & & & & Tirar baño & \\
\hline \multicolumn{6}{|c|}{ Secar al aire, en superficie plana } \\
\hline & & Ablandar a ma & & & \\
\hline
\end{tabular}

Tabla 1. Planilla de proceso de curtido. 


\section{Determinación de propiedades físicas}

Para caracterizar los cueros obtenidos se realizaron ensayos de resistencia a la tracción, resistencia al desgarro y temperatura de contracción, para lo cual fueron cortadas, con troqueles adecuados, 10 probetas para cada tipo de análisis. En la Figura 2 se observan las probetas de cada tipo de ensayo.

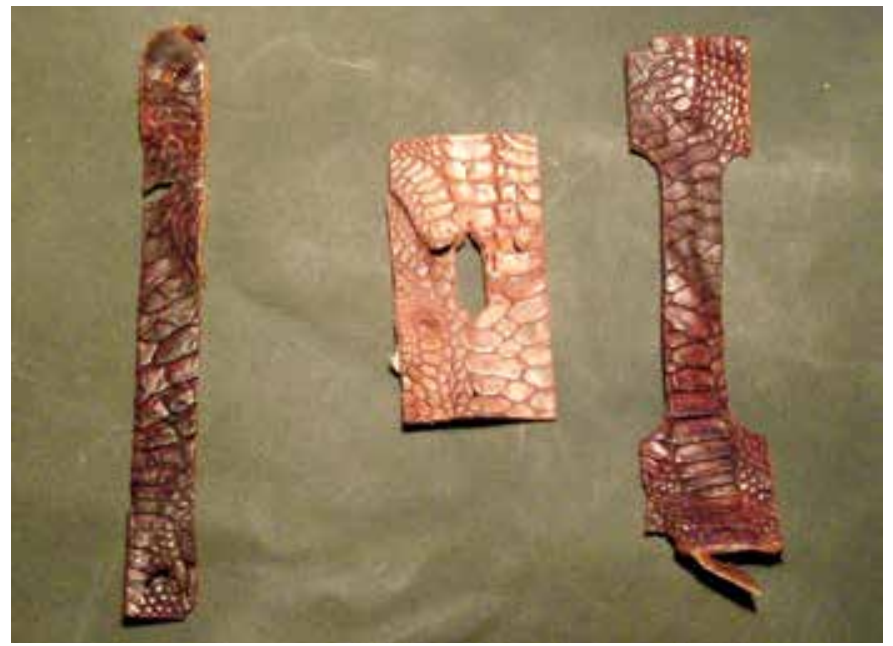

Figura 2. Probetas de ensayo.

La temperatura de contracción se midió en un equipo SATRA modelo STD 114, que consiste en un baño de agua cerrado, con un termómetro, en el que se coloca una muestra de cuero, de $100 \mathrm{~mm}$ de largo y $10 \mathrm{~mm}$ de ancho. Se calentó el baño con un mechero en forma progresiva y se tomó como temperatura de contracción la correspondiente al momento en que comenzó a contraerse el cuero.

Para la determinación de la resistencia al desgarro y la resistencia a la tracción se utilizó un dinamómetro Instron 1130 equipado con una celda de $50 \mathrm{~kg}$. Para medir el espesor de las probetas de ensayo se usó un medidor de espesor de cuero MAQTEST. Estos equipos cumplen los requisitos de las normas utilizadas para realizar los ensayos.

La resistencia al desgarro se determinó de acuerdo a la norma ISO 3377-2:2002. Las probetas fueron cortadas y medido a cada una su espesor, según la norma. Fueron sometidas a un esfuerzo de desgarro en el dinamómetro, a una velocidad de separación de mordazas constante, de $100 \mathrm{~mm} / \mathrm{s}$. Se registró el valor máximo de carga y con el espesor medido se calculó el valor de resistencia al desgarro.
Para la determinación de la resistencia a la tracción se utilizó la norma ISO 3376:2002. Se cortaron las probetas y se midió su espesor. En el dinamómetro se determinó para cada probeta la carga de ruptura, a una velocidad de separación de mordazas de $100 \mathrm{~mm} / \mathrm{s}$. Con el valor de la carga de ruptura, el espesor medido de cada probeta y el ancho de probeta se calculó su resistencia a la tracción.

Los resultados de resistencia a la tracción y al desgarro se compararon con valores requeridos habitualmente para distintos usos.

\section{Productos}

Con los cueros obtenidos se confeccionaron artículos de marroquinería, como cinturones, billeteras y porta celulares (Figura 3). No se presentaron dificultades para la manipulación ni para la confección de los productos.

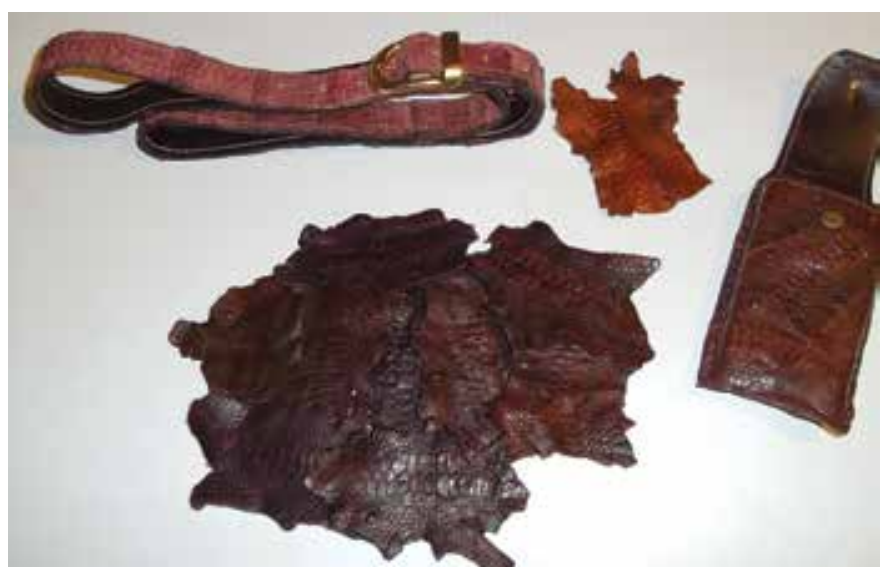

Figura 3. Artículos en cuero de pata de gallina.

\section{Resultados}

En la Tablas 2 a 4 se presentan los datos obtenidos de tracción a la ruptura, resistencia al desgarro y temperatura de contracción de los cueros obtenidos en el proceso de curtido.

En la Tabla 5 se comparan los valores de tracción y desgarro de los cueros de pata de gallina con valores habituales para distintos tipos de uso de los cueros.

\begin{tabular}{|c|c|c|c|c|}
\hline \multicolumn{5}{|c|}{ RESISTENCIA A LA TRACCIÓN A LA RUPTURA } \\
\hline Probeta & $\begin{array}{c}\text { Espesor Probeta } \\
(\mathbf{m m})\end{array}$ & $\begin{array}{c}\text { Ancho Probeta } \\
(\mathbf{m m})\end{array}$ & $\begin{array}{c}\text { Carga } \\
(\mathbf{k g})\end{array}$ & $\begin{array}{c}\text { Tracción } \\
\left(\mathbf{k g} / \mathbf{m m}^{2}\right)\end{array}$ \\
\hline $\mathbf{1}$ & 1,12 & 10 & 5,02 & 0,45 \\
\hline $\mathbf{2}$ & 1,07 & 10 & 5,47 & 0,51 \\
\hline $\mathbf{3}$ & 1,39 & 10 & 7,03 & 0,51 \\
\hline $\mathbf{4}$ & 0,65 & 10 & 10,63 & 0,67 \\
\hline $\mathbf{5}$ & 1,30 & 10 & 8,77 & 0,64 \\
\hline $\mathbf{6}$ & 1,08 & 10 & 7,10 & 0,55 \\
\hline $\mathbf{7}$ & 0,89 & 10 & 4,90 & 0,54 \\
\hline $\mathbf{8}$ & 0,91 & 10 & 4,94 & 0,63 \\
\hline $\mathbf{9}$ & 0,72 & 10 & 4,50 & 0,38 \\
\hline $\mathbf{1 0}$ & 1,01 & 10 & 3,80 & 0,65 \\
\hline
\end{tabular}

Tabla 2. Valores de tracción. 


\begin{tabular}{|c|c|c|c|}
\hline \multicolumn{4}{|c|}{ RESISTENCIA AL DESGARRO } \\
\hline Probeta & $\begin{array}{c}\text { Espesor Probeta } \\
(\mathbf{m m})\end{array}$ & $\begin{array}{c}\text { Carga } \\
\mathbf{( k g})\end{array}$ & $\begin{array}{c}\text { Desgarro } \\
(\mathbf{k g} / \mathbf{m m})\end{array}$ \\
\hline $\mathbf{1}$ & 1,32 & 2,51 & 1,90 \\
\hline $\mathbf{2}$ & 1,09 & 2,18 & 2,00 \\
\hline $\mathbf{3}$ & 1,08 & 2,00 & 1,85 \\
\hline $\mathbf{4}$ & 1,27 & 2,84 & 2,24 \\
\hline $\mathbf{5}$ & 1,20 & 2,00 & 1,67 \\
\hline $\mathbf{6}$ & 1,06 & 1,35 & 1,27 \\
\hline $\mathbf{7}$ & 0,95 & 3,71 & 3,90 \\
\hline $\mathbf{8}$ & 0,86 & 1,64 & 1,91 \\
\hline $\mathbf{9}$ & 0,77 & 1,71 & 2,22 \\
\hline $\mathbf{1 0}$ & 0,98 & 1,96 & 2,00 \\
\hline
\end{tabular}

Tabla 3. Resistencia al desgarro.

\begin{tabular}{|c|c|}
\hline Probeta & $\begin{array}{c}\text { Temperatura de contracción } \\
\left({ }^{\mathbf{C}} \mathbf{C}\right)\end{array}$ \\
\hline $\mathbf{1}$ & 80 \\
\hline $\mathbf{2}$ & 80 \\
\hline $\mathbf{3}$ & 79 \\
\hline $\mathbf{4}$ & 80 \\
\hline $\mathbf{5}$ & 80 \\
\hline $\mathbf{6}$ & 80 \\
\hline $\mathbf{7}$ & 79 \\
\hline $\mathbf{8}$ & 80 \\
\hline $\mathbf{9}$ & 79 \\
\hline $\mathbf{1 0}$ & 80 \\
\hline PROMEDIO & 80 \\
\hline
\end{tabular}

Tabla 4. Temperatura de contracción.

\begin{tabular}{|c|c|c|c|c|}
\hline \multicolumn{5}{|c|}{ RESISTENCIA AL DESGARRO } \\
\hline Ensayo & $\begin{array}{c}\text { Cuero pata } \\
\text { de gallina }\end{array}$ & $\begin{array}{c}\text { Cuero para } \\
\text { capellada }\end{array}$ & $\begin{array}{c}\text { Cuero para } \\
\text { forro de calzado }\end{array}$ & $\begin{array}{c}\text { Cuero marroquinería } \\
\text { curtido vegetal }\end{array}$ \\
\hline $\begin{array}{c}\text { Resistencia } \\
\text { a la tracción } \\
\left(\mathbf{k g} / \mathbf{m m}^{\mathbf{2}}\right)\end{array}$ & 0,7 & 2,0 & 1,5 & 1,0 \\
\hline $\begin{array}{c}\text { Resistencia } \\
\text { al desgarro } \\
\text { (kg/mm) }\end{array}$ & 2,1 & 4,0 & 1,5 & 1,0 \\
\hline
\end{tabular}

Tabla 5. Comparación con especificaciones para distintos tipos de cueros.

\section{Discusión}

Los valores de temperatura de contracción obtenidos con este proceso de curtido vegetal de pieles de patas de gallina, de $80{ }^{\circ} \mathrm{C}$ según la Tabla 4, muestran que el colágeno fue estabilizado por el proceso de curtido, ya que es mayor a la temperatura de contracción de las pieles crudas. Este valor de $80^{\circ} \mathrm{C}$ es inferior a los que se obtienen en un cuero curtido al cromo, en el que pueden alcanzar valores de $100^{\circ} \mathrm{C}$ a $115^{\circ} \mathrm{C}$, pero es comparable con los valores de temperatura de contracción de cueros curtidos con taninos vegetales, glutaraldehído, aluminio o zirconio, que se encuentran entre los $70^{\circ} \mathrm{C}$ y $80^{\circ} \mathrm{C}$.

La resistencia a la tracción dio resultados inferiores a los requeridos habitualmente para los tipos de artículos que se indican en la Tabla 5. Los valores de resistencia al desgarro de los cueros de pata de gallina son menores al valor requerido para la confección de capellada de calzado, pero son superiores a los necesarios para la confección de otros productos, como forro de calzado o artículos de marroquinería. Estos valores bajos pueden deberse a que los agentes de engrase utilizados en el recurtido no penetraron o fueron insuficientes, dado que su función es lubricar las fibras del colágeno en el cuero para lograr las propiedades de tracción y desgarro adecuadas. También puede deberse a que los taninos vegetales utilizados en la etapa de curtido o la combinación utilizada no fueron los apropiados para lograr mejores resultados en esas propiedades.

Pese a estos resultados bajos de resistencia a la tracción y al desgarro, no se presentaron dificultades durante la confección de los artículos que fueron fabricados con estos cueros (Figura 2). Estos también podrían ser utilizados en otros productos, como calzado o vestimenta, que aunque resulten más exigentes en cuanto a esos requerimientos, admiten su incorporación como apliques.

\section{Conclusiones}

Este proceso de curtido vegetal para pieles de pata de gallina fue efectivo para su transformación en cuero, según lo marcan los valores de temperatura de contracción alcanzados.

Los resultados bajos de resistencia a la tracción y al desgarro son factibles de ser mejorados haciendo modificaciones en la etapa de curtido, ya sea en los tipos como en las cantidades de taninos utilizados. Pero es en la etapa del recurtido en la que las variaciones en cantidades y tipos de taninos, engrases, más el agregado de recurtientes sintéticos pueden tener mayores efectos en la mejora de esas propiedades.

Los cueros obtenidos con la piel de las patas de gallina suponen un agregado de valor a un subproducto de la faena de aves. Este agregado está dado por los productos que se pueden fabricar con ellos, que por su apariencia similar a los cueros de reptil pueden ser considerados exóticos y de alto precio en el mercado.

El proceso que se utilizó para hacer este curtido, sin sulfuro de sodio y sin sales de cromo, permite que pueda replicarse por medios artesanales y en pequeñas cantidades, ya que se eliminaron los productos que pueden ocasionar mayores dificultades para un correcto manejo medioambiental.

\section{Reconocimientos}

Agradecemos a la Dra. Karina Llorente y a la firma Avícola Tenent por el suministro de las patas de gallina.

Al Sr. Marcelo De Simone, quien confeccionó los productos en cuero.

A la estudiante Cecilia Pereira, quien tuvo a su cargo la engorrosa tarea de pelar las patas de gallina. 


\section{Referencias}

- ADZET ADZET, J. M. Química-técnica de tenería. Barcelona: Romanyá/Valls, 1985. ISBN: 84-398-3375-x

- BASF. Vademécum para el técnico en curtición. $3^{\circ} \mathrm{ed}$. [s.1.]: BASF, 1995.

- CLARA, M. Aves [En línea]. Montevideo: Facultad de Ciencias, 2000. [Consulta: 12 de junio de 2012]. Disponible en: http://zvert. fcien.edu.uy/avesrep.doc

- INTERNATIONAL STANDARD ORGANIZATION (Switzerland). ISO 3376 (IULTCS/IUP 6): Leather. Physical and mechanical tests. Determination of tensile strength and percentage extension. Geneva: ISO, 2002.

- INTERNATIONAL STANDARD ORGANIZATION (Switzerland). ISO 3377-2 (IULTCS/IUP 8): Leather. Physical and mechanical tests. Determination of tear load Part 2: Double edge tear. Geneva: ISO, 2002.

- MOREIRA, M. V.; TEIXEIRA, R.C. Estado da arte tecnológico em processamento do couro: revisão bibliográfica no âmbito internacional. Porto Alegre: Centro Nacional de Tecnologias Limpas, 2003

- SATRA TECHNOLOGY CENTRE. Satra TM17 shrinkage temperature of leather. Kettering: SATRA, 2012.

- Páginas web: Cueronet - http://www.cueronet.com/ 